\title{
Microbiome Populations in Lebanese Slaughterhouse Settings
}

\author{
Prattis SM ${ }^{1 *}$, Shaib HA ${ }^{1}$, Zgheib ${ }^{2}$, Raad $A^{3}$, Hassan $\mathrm{ZA}^{3}$, and Rifai $\mathbf{R}^{\mathbf{1}}$ \\ ${ }^{1}$ Department of Agriculture, American University of Beirut, Beirut, Lebanon \\ ${ }^{2}$ Department of Nutrition, American University of Beirut, Beirut, Lebanon \\ ${ }^{3}$ Supervising Veterinarian, Northern District Bekka'a Valley, Ministry of Agriculture, Lebanon
}

Received: October 10, 2014; Accepted: February 25, 2015; Published: March 10, 2015

*Corresponding author: Prattis SM, Department of Agriculture, American University of Beirut, Beirut Lebanon, Tel: +961-01-340460 Ext: 4451; Fax: +961-01-744460; E-mail: sp07@aub.edu.lb

\begin{abstract}
Lebanon has traditionally relied on small ruminants and cattle livestock production to generate meat and dairy food products for the region. Consumer interest and changing residential populations have increased demand for these products, and highlighted food safety concerns along the community chain. Traditional livestock production levels have been unable to meet population demand for meat, necessitating supplemental importation of Brazilian and European cattle for this purpose. While microbial assessment has been studied in dairy and poultry sector products and retail fast foods there are no published reports examining microbial populations in slaughterhouse environments or on cut raw red meat in Lebanon. We determined total aerobic bacterial counts per gram of minced muscle specimen and aerobic bacterial counts per Replicate Organism Detection and Counting Plates (RODAC) plate surfaces (using in-house constructed plates) to characterize microbial species populations found in cattle and sheep carcasses immediately after halal slaughter, and on facility floors and walls within two main Lebanese slaughterhouses located in urban Beirut and rural Baalbek, the Bekaa valley, respectively. We also examined the histological appearance of raw meat muscle using snap frozen Optimal Cutting Temperature (OCT) media-embedded muscle specimens placed in liquid nitrogen cooled isopentane. The most frequently isolated bacterial species were Staphylococcus aureus and Yersinia pseudotuberculosis. Statistical examination of microbial number comparisons including both sheep and cattle microbial counts from initial individual visits made to both slaughterhouses were not significantly different using individual Student's t Tests. However, Chi Square comparison of separate cattle and sheep muscle microbial counts obtained at initial visits to the Beirut and Baalbek slaughterhouses evaluated at initial culture testing were highly significantly different to the $90^{\text {th }}$ percent probability level $(\chi 2=3.4225$, degrees of freedom $=1$, alpha 0.10 $=2.706$ ). This is the first published study of this type in Lebanon. Examining aerobic microbial species and processing procedures exhibited in the slaughterhouse environment provides a basis upon which to assess current food safety preventive practices. Developing a surveillance testing system for food animal health and zoonotic disease should facilitate and to consider future Hazard Analysis and Critical Control Point (HACCP) planning critical control point sites for intervention within the Lebanese setting.
\end{abstract}

Keywords: Muscle; Aerobic plate counts; Zoonoses; Slaughter house; Food microbiology; Meat processing; HACCP; Lebanon

\section{Introduction}

Lebanon has had a long history of sustained crop and animal agricultural production based predominantly in the heart of the Bekaa Valley [1]. Domestic animal production in Lebanon has historically consisted of domestic poultry, lamb and mutton, fish, goat and beef meat production with ongoing emphasis on diary production for large commercial milk, cheese and labneh product markets especially [1,2]. Historically farmers, consumers and producers have focused chiefly on individual herds producing lamb and goats, complementing well-developed poultry meat and egg markets, and several large cattle dairy producers developing products in support of the large Lebanese domestic dairy industry. However, in Lebanon there has been an increased demand for meat products that domestic markets have not met $[1,2]$. Unlike lamb and goat meat products, most beef in Lebanon is obtained following importation from South America and Europe [1-3]. Increased consumer demands have highlighted food safety concerns in the red meat value chain. Further, the presence of regional hostilities has resulted in human relocation possibly accompanied by domestic animal herds (FAO Lebanon, individual communication), with somewhat porous boundaries especially in mountainous regions surrounding the Bekaa valley. Syrian and Lebanese animal farmers have traditionally and repeatedly crossed the border in search of grazing lands, medicines, and limited levels of veterinary care [2]. Trans boundary disease transmission and disease susceptibility among unprotected herds may be increased [3]. Examination of carcasses during local routine slaughter practices can determine if there are negative impacts on animal and human health, identify microbial species and prevent introduction of food borne illness into the food chain [3].

There are no published reports examining red meat Microbiome at the point of origin into the food chain in slaughterhouse settings within Lebanon. Few studies have examined meat products present in the food chain in Lebanon. None have evaluated red meat carcasses or slaughterhouse environments, including evaluation of two main slaughterhouses processing meat products for Beirut and inner suburbs, and 
the Bekaa Valley respectively, for environmental and product microbial populations. These populations can be assessed as quality indicators that might contribute to the marketing meat products in more upscale settings, and/or decreasing the incidence of food - borne illnesses experienced by consumers in this region. Pathogenic microbial populations associated with vegetables have been well described elsewhere [4]. Microbial populations and antibiotic resistance in animal food products in both retail and wholesale settings have been examined in the Middle East and Africa through evaluation of heritage cheeses and dairy products $[5,6]$ and meat $[7,8]$. However, fewer such studies have been performed at various steps of the regional meat food chain. One investigator isolated microbial populations from meat - based fast foods in Lebanon and identified a range of indicator and pathogenic bacteria contaminants that were present in both Shawarma, a rotating spit of typically lamb meat that is sliced to make pita bread sandwiches with dressing and/ or vegetables, and Lahm bi Ajeen, a meat - based flat meat pie, with slight differences in regional distribution and contamination level [9]. These microbial populations included Escherichia coli, and other pathogenic coliform species, Salmonella species, and pathogenic Staphylococcus aureus [9].

Currently there are no central regulations or standards governing oversight of meat production, identification and traceability at all steps along the process from livestock production farms through retail and restaurant products and services in Lebanon [10,11]. Responsibility for this process is shared through the efforts of several different Ministerial organizations. Reinforcement of existing laws and guidelines can be quite variable $[10,11]$. Cultural practices in Lebanon include foods that could be easily compromised by microbial contamination, including special preparations of raw liver (Lahme nayi) and raw meat, typically lamb and mutton (Kebbi nayi) [9]. It would be advantageous to determine baseline levels and types of microbial species typically found in slaughterhouse environments. These studies serve as a first step towards developing pathogen reduction processes through using Hazard Analysis and Critical Control Point (HACCP) systems (FSIS 1996) within the Lebanese political context and governmental systems.

There is increasing interest in determining the "Microbiome" of a given system, defined as the collection of microbial species and interactions that are characteristically found in different organisms, locations and settings [12,13]. Assessing the presence of microbial strains in the slaughterhouse environment that have been previously associated with foodborne illness in people is a first step in developing a more robust food safety surveillance system. The best food surveillance systems enhance food safety for consumers and increase value for meat and meat byproducts in the marketplace. This study is the first one to evaluate the presence of microbial species in Lebanese slaughterhouses, thus defining an initial slaughterhouse Microbiome in Lebanon. We conducted an initial study to determine aerobic and anaerobic bacteria counts in meat specimens and through use of in house constructed Replicate Organism Detection and Counting (RODAC) plates to evaluate selected floor and wall sites within Lebanese slaughterhouses.

\section{Methods \\ Specimen collection}

An overall experimental population of thirty seven individual animals was examined, with one to three muscle samples obtained per animal. All animals processed by each facility were privately owned and were subsequently sold on site to permitted wholesalers, small meat markets and local supermarkets, who provided their own transportation and staffing. Cooling, chilling, or hot water disinfection carcass facilities were not available at either slaughterhouse facility.

Microbiological specimens were collected as detailed below form individual muscles with animal carcasses, and form facility floors and walls, separately. Specimens were taken during period of active slaughtering activity for cattle and small ruminants. Two visits were made to the Beirut slaughterhouse facility and three to the Baalbek slaughterhouse facility. An overall experimental population of thirty seven individual animals was examined, with one to two muscle specimens obtained per animal. An attempt was made to take specimens from similar anatomic muscle locations in successive individual animals and study visits, in consultation with standard anatomy texts $[14,15]$ and within the restrictions imposed by animal stock owners and slaughterhouse workers. Microbial and meat muscle samples were obtained from freshly slaughtered animals ("hot carcasses", [16,17]) using sterilized instruments. Sheep and cattle were slaughtered using traditional halal methods by slaughterhouse employees who were members of the Syndicate (Union) of Slaughterhouse Workers. Cattle presenting for slaughter were predominantly Brazilian Brahmin and Brahmin - Holstein/ Friesian cross imports, with local resident cattle presumably raised in Lebanon and several European imported cattle. Both male and female animals were represented among the population housed, observed and selected for slaughter and were included without any differentiation in the study. Definitive information regarding the age of each animal was not provided to us and was not known in all cases. Small ruminants presenting for slaughter were predominantly fat tail Awassi sheep and Baladi goat crossbred individuals that are characteristic of regional breeds found locally in Lebanon $[1,3]$.

Whole carcasses were subdivided into primal cuts, with separation of intact viscera, pluck (tongue, trachea and esophagus, heart, lungs, and liver), head and hooves prior to specimen collection. As each animal was individually owned we were restricted from sampling commercially valuable muscle bundles. Accordingly, sample selection sites in the thorax and medial forelimb included the diaphragm, subscapularis muscle; infraspinatus and teres major muscles, and trapezius muscle. Medial hind limb sample sites included sartorius, pectineus and vastus lateralis muscles. Individual muscles were identified and recorded in the slaughterhouse immediately after slaughter and sampling. Each muscle specimen was placed in a sterile Petri dish, wrapped with parafilm (Menasha, WI 54952, USA) and placed on a bed of ice. The specimens were transported on ice to the Animal and Veterinary Science Laboratory at the American University of Beirut for further processing. 
Individual environmental sampling plates were used to obtain samples of microbial populations on the floors and walls of the Beirut and Bekaa slaughterhouses. Petri plates containing raised agar in 6 millimeter diameter small Petri dishes nested into larger 10 millimeter Petri plates (see below for descriptions of construction) were pressed against walls and floor sites, placed on ice, and transported to the laboratory for further growth and biochemical analysis using API 20E Enterobacteriaceae strips and Staphylococcus determination methods (BioMérieux S.A 69280 Marcy-l' Etoile, France).

\section{Specimen processing}

Histological Evaluation: Muscle samples were trimmed of visible fat and fascia using sterile forceps and fine scissors. Individual $6 \mathrm{~mm}$ sterile biopsy punches (Miltex, Inc. York, PA 17402 , USA) were used to sample a total of three biopsies per cleaned muscle, which were then oriented and were put as a group into OCT medium (Jung, Leica Instruments, Gmbh, Nussloch Germany) enclosed in aluminum foil cups and snap - frozen in Isopentane (Lot number STBC9004V, Sigma-Aldrich) cooled in liquid Nitrogen $\left(-156^{\circ} \mathrm{C}\right)$. These blocks were wrapped and kept at $-80^{\circ} \mathrm{C}$ until further processed using cryostat sectioning. The frozen muscle biopsies were cut into 7 micrometer thick sections using a Leica cryostat set at $-30^{\circ} \mathrm{C}$ (Leica CM1850, Leica Instruments Gmbh, Nussloch, Germany). Three cryostat sections were fixed onto a charged slide pre warmed to room temperature for at least 10 minutes and were then stained in Harris' hematoxylin for 45 seconds, rinsed in tap water then placed into a $0.5 \%$ acid alcohol solution several times to remove excess stain. The slides were then rinsed in tap water followed by Scott's tap water substitute to enhance the blue coloration. Following a second tap water rinse the slides were then counterstained in 1\% Eosin Y (Sigma Aldrich, St. Louis MO, USA Lot number SLBG9284) for 15 seconds. Finally, the muscle sections were dehydrated through a series of graded alcohols to $96 \%$ ethanol, cleared in xylene and mounted in Permount (Fisher Scientific, NJ, and USA). Histopathological sections were screened for normal muscle histological appearance using routine microscopic observation (Leica DMLS, Nussloch, Germany).

\section{Microbiological culture preparation-muscle}

Muscle samples of 9-15 g average weight were placed into new sterile Petri dishes and were aseptically minced into fine pieces using sterile scissors and scalpels, soaked in $20 \mathrm{ml}$ saline at room temperature for 20 minutes, then agitated for 2 minutes and transferred into sterile $50 \mathrm{ml}$ tubes. Twenty five micro liters of the minced muscle supernatant was then spread across the surface of a Petri plate containing Nutrient Agar $(2.8 \%$ in water), containing peptic digest of animal tissue, beef extract, yeast extract, sodium chloride and agar (Himedia laboratories Pvt, Limited, Mumbai - 400 086, India) for determination of total bacterial Aerobic count (Aerobic Plate Count, APC; colony forming units, cfu). A separate loopful of supernatant streak inoculation was used for bacterial isolation onto each of the following plates: 1) MacConkey agar (Oxoid Ltd, Hampshire, $\mathrm{UK})$ to differentiate lactose from non-lactose fermenter gram negative bacteria; 2) Brilliant Green Agar (Oxoid Ltd, Hampshire UK) for isolation of suspected Salmonella pathogenic species; 3) Slanetz Bartley Agar (Scharlau, Scharlab, S.L., Spain) for isolation of suspected Streptococcus species, and 4) Mannitol Salt Agar (Oxoid Ltd., Hampshire, UK) for isolation of Staphylococcus aureus. Subcultures were incubated at $37^{\circ} \mathrm{C}$ overnight. Bacterial inoculants were visually observed sequentially after 20 hours incubation. Isolated colonies were grown over Triple Sugar Iron Agar (TSI), and used for gram staining and bacterial identification. These bacterial colonies were isolated; gram stained with Crystal Violet, Lugol's Iodine (Potassium Iodine, The British Dry House, and London, UK) and Safranin (The British Dry House, London, UK) using routine methods [Gram 1884] and observed microscopically (Leica DMLS, Nussloch, Germany) under the oil immersion power (1000X).

For further identification of isolated gram stained and characterized bacterial colonies, lactose and nonlactose fermenting bacterial isolates were speciated using previously published schematic methods [18] and the API 20E kit (BioMérieux, Inc, Marcy-L' Etoile, France), following manufacturers direction. Final determination of bacterial isolate characteristics was obtained using the API $20 \mathrm{E}$ reactivity and assessment of database of bacterial strain characteristic profiles. The following biochemical reactions were assessed: Orthonitrophenyl-Beta-galactosidase, Lysine Decarboxylase, Citrate Decarboxylase, $\mathrm{H}_{2} \mathrm{~S}$ production, Urease production, Tryptophan Deaminase, the Indole test, the Voges-Proskauer test, and Gelatinase, Glucose, Mannitol, Inositol, Sorbitol, Rhamnose, Sucrose, Melibiose, Amylose, Arabinose and Oxidase test. Suspected Streptococcus species isolates that had grown over Slanetz Bartley selective medium were initially confirmed to the genus level. These results were then confirmed using previously published schematic approaches that evaluated for biochemical results following L-Arabinose, Arginine Dihydrolase and Mannitol testing profile reactivity $[19,20]$.

\section{Environmental Microbiology - Slaughterhouses}

We developed an in-house version of commercially available [Figure 1] RODAC plates using two Petri dishes sized 6 and 10 $\mathrm{mm}$ of diameter that were glued to each other using cold acetone $\left(-80^{\circ} \mathrm{C}\right)$ (JRZ Plastilab, Lebanon). Fifteen milliliters of Nutrient and other agars as described above were poured into the smaller 6 milliliter diameter but taller plate, which was then attached to a larger 10 milliliter diameter Petri dish base, enabling it to extend above the rim of the larger Petri plate and therefore simulating a commercially purchased RODAC plate assembly. These sterile assemblies were then pressed against either the floor or wall of the slaughterhouse in locations where animals entered the facilities and were routinely slaughtered while being handled by slaughterhouse workers [Figures 2,3]. Separate floor and wall sampling sites for both slaughterhouse facilities are illustrated in Figures 4 and 5. Microbial RODAC plate and muscle sampling occurred during slaughtering periods scheduled for either early in the morning (Bekaa valley), or late evening (Beirut). Specimens were placed on ice for transit back to the laboratory, where muscle specimens were processed as noted above, and 


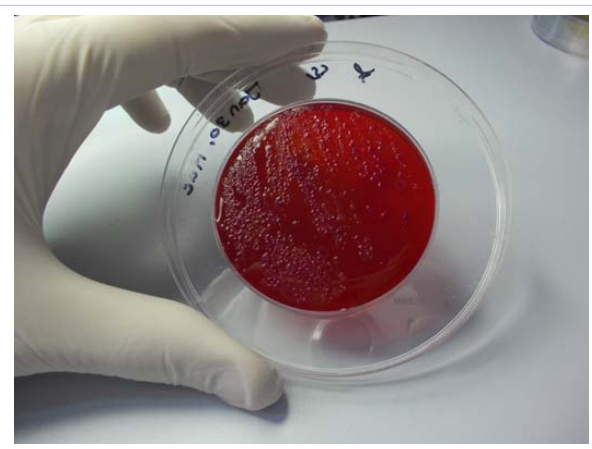

Figure 1: Representative in house RODAC culture from slaughterhouse facilities.

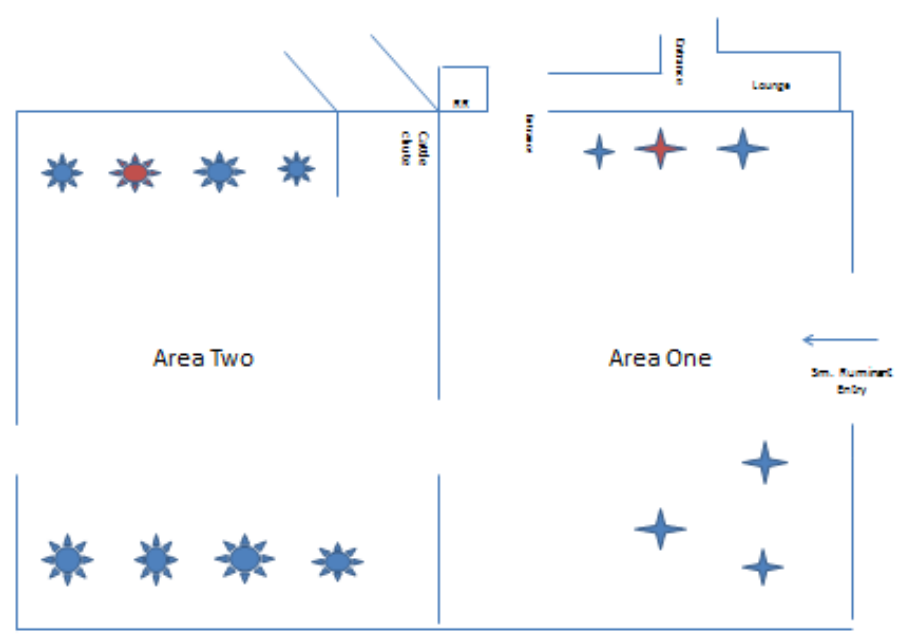

Figure 2: Schematic of Baalbek slaughterhouse illustrating RODAC specimen sampling locations, Broken lines indicate animal and human egress locations while crosses indicate animal carcass stations. Small ruminants were slaughtered in Area one while cattle were slaughtered in Area two. Cattle chute was closed until animal entered. Sampling sites are indicated by filled star sites. RR = Restroom and sinks.

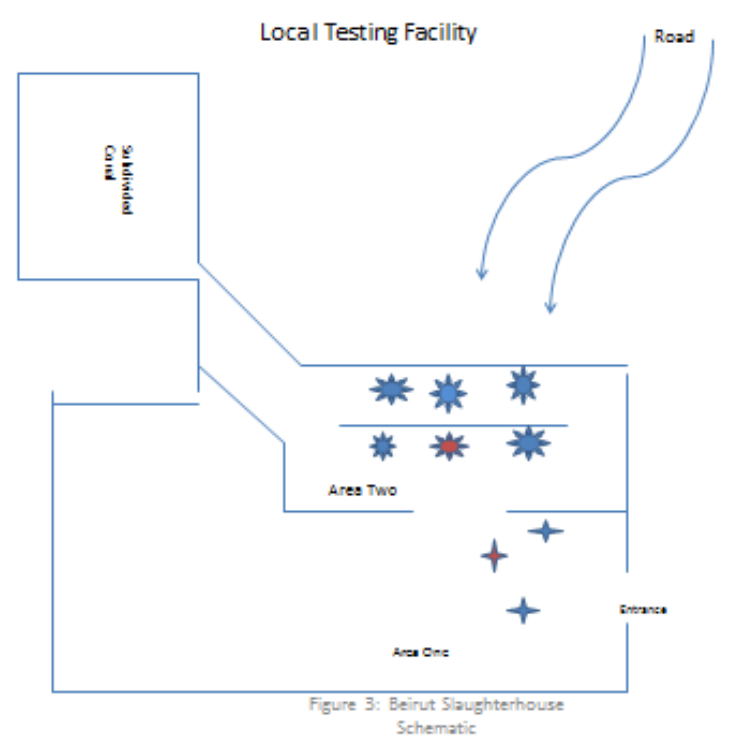

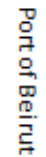

Figure 3: Schematic of Beirut slaughterhouse illustrating RODAC specimen sampling locations, broken lines indicate animal and human egress locations while crosses indicate animal carcass stations. Small ruminants were slaughtered in Area 1 while cattle were slaughtered in Area 2. Sampling sites are indicated by filled cross sites. 
RODAC plate specimens were initially incubated at $37^{\circ} \mathrm{C}$ in $5 \%$ oxygen conditions. Individual plates were sub cultured, isolated, enumerated and cultured for biochemical determination as noted above for Muscle Microbial Supernatant Culture methods.

\section{Results}

\section{Histological appearance}

Muscle histological cryostat sections obtained from cattle and sheep at slaughter revealed normal skeletal muscle micro anatomical appearance with hematoxylin and eosin staining in most cases [Figure 4]. There were also putative parasitic structures evident in domestic lamb muscle using this method (Prattis et al, in preparation).

\section{Muscle supernatant culture results}

We isolated and identified a relatively narrow spectrum of organisms from the Baalbek and Beirut Slaughterhouse Facilities [Tables 1-3 and Figure 5]. Cattle muscle tissues contained high numbers of Staphylococcus aureus and Yersinia pseudotuberculosis organisms. Klebsiella oxytoca was detected in sheep at levels comparable to those found in cattle. Overall, sheep exhibited lower numbers of total aerobic count per gram of muscle for Escherichia coli and Staphylococcus aureus, among other microbial species [Table 1]. These included Staphylococcus aureus, Yersinia pseudo tuberculosis, and Escherichia coli from animals slaughtered in the Baalbek Slaughterhouse Facility, and Pasteurella pneumonia / haemolytica, Klebsiella oxytoca, Yersinia enterocolitica and non differentiated Pantoea species from the Beirut Slaughterhouse Facility for the first visit, followed by Escherichia coli, Staphylococcus aureus, Klebsiella oxytoca, Proteus vulgaris and Serratia plymuthica during the second visit [Table 1]. Considering both facilities together determined that the most frequently identified agent in muscle specimens was Staphylococcus aureus (31.58 \%) followed by Yersinia pseudo tuberculosis (18.42\%). A sizable group of microbes were not identified to the species level using classical microbial culture and biochemical assay phenotyping techniques and were classified as non-Identified species (15.79\%). Proteus vulgaris $(7.89 \%)$ and Klebsiella oxytoca (7.89\%) were frequently identified, as were Serratia plymuthica and Streptococcus species (both 2.63 $\%$ ) [Figure 5]. In one case we were unable to subculture the isolate over TSI media and so did not perform any biochemical characterization (Non applicable, $2.63 \%$ ) [Figure 5].

A subset of the data from the first visits to each facility provides a direct comparison between the two slaughterhouse sites based on typical operative procedures. This period was chosen for closer examination because slaughterhouse workers appeared to intensify facility cleaning efforts after our first data collection visits to each location. Enumerating muscle specimen supernatant cultures on our first visit to the Beirut slaughterhouse facility demonstrated that it was not unusual to identify 100 colonies per muscle specimens for bacterial species including Pasteurella pneumonia/ haemolytica (11.11\%), Klebsiella oxytoca (22.22\%), Yersinia enterocolitica (11.11\%) and Pantoea species (11.11\%). Several microbial organisms were present at high average counts including Pantoea species at 332.5 average bacterial numbers per $\mathrm{cm}^{2}$, and Chryseomonas luteola at 110.0 average bacterial numbers per $\mathrm{cm}^{2}$ of muscle sampled [Table 2].

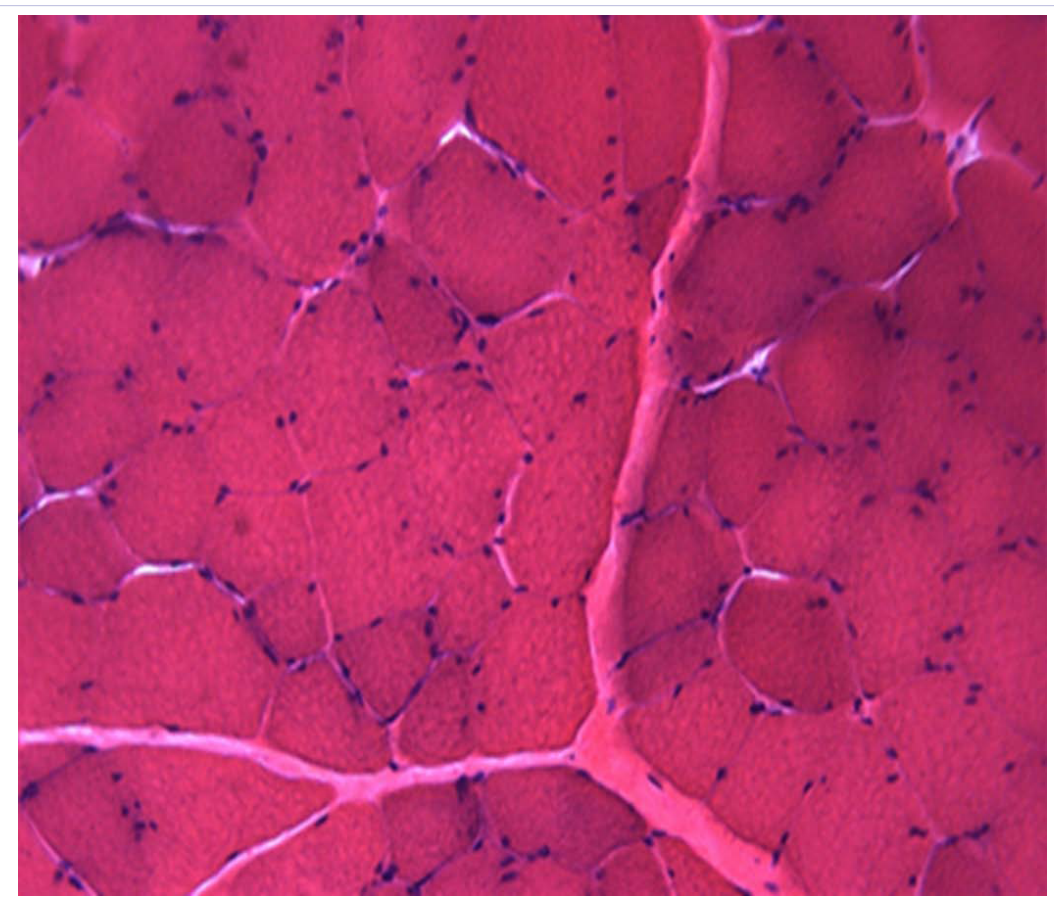

Figure 4: Cryostat Sectioned Muscle Histology. Histological appearance of freshly slaughtered muscle specimens, Representative normal bovine skeletal muscle obtained at slaughter is shown above. Tissues were flash frozen; cryostat sectioned and stained using hematoxylin and eosin. $200 \mathrm{x}$ magnifications. 
Table 1: Raw Muscle aerobic plate counts following Slaughterhouse Collection.

\begin{tabular}{|c|c|c|c|c|}
\hline Facility & Origin & Species & Muscle Region & Aerobic Count \\
\hline \multicolumn{5}{|l|}{ Bekaa } \\
\hline 1 & Lebanon & Sheep & Diaphragm & 2823.01 \\
\hline 2 & Lebanon & Sheep & Forelimb & 1563.51 \\
\hline 3 & Brazil & Cattle & Diaphragm & 2388.7 \\
\hline 4 & Brazil & Cattle & Forelimb & 998.91 \\
\hline 5 & Brazil & Cattle & Hindlimb & 4039.08 \\
\hline 6 & Local Bekaa & Cattle & Scapula & 521.17 \\
\hline 7 & Local Bekaa & Cattle & Scapula & 347.44 \\
\hline 8 & Local Bekaa & Cattle & Diaphragm & 781.75 \\
\hline 9 & Local Bekaa & Cattle & Hindlimb & 912.05 \\
\hline \multicolumn{5}{|l|}{ Beirut } \\
\hline \multicolumn{5}{|l|}{$1^{\text {st }}$ Visit } \\
\hline 10 & Lebanon & Sheep & Diaphragm & 16371.3 \\
\hline 11 & Lebanon & Sheep & Forelimb & 5878.31 \\
\hline 12 & Brazil & Cattle & Diaphragm & 8.9 \\
\hline 13 & Local Bekaa & Cattle & Forelimb & 1548.29 \\
\hline 14 & Brazil & Cattle & Hindlimb & 1792.04 \\
\hline 15 & Brazil & Cattle & Scapula & 62497.37 \\
\hline 16 & Brazil & Cattle & Scapula & 5206.64 \\
\hline 17 & Europe & Cattle & Diaphragm & 1513.31 \\
\hline 18 & European & Cattle & Hindlimb & 230.05 \\
\hline \multicolumn{5}{|l|}{$2^{\text {nd }}$ Visit } \\
\hline 19 & Lebanon & Sheep & Diaphragm & 181.4 \\
\hline 20 & Lebanon & Sheep & Forelimb & 824.96 \\
\hline 21 & Brazil & Cattle & Diaphragm & 107.41 \\
\hline 22 & Local Bekaa & Cattle & Forelimb & 2744.47 \\
\hline 23 & Brazil & Cattle & Hind limb & 3239.42 \\
\hline 24 & Brazil & Cattle & Scapula & 3782.81 \\
\hline 25 & Brazil & Cattle & Scapula & 3466.98 \\
\hline 26 & $\mathrm{~N} / \mathrm{A}$ & $\mathrm{N} / \mathrm{A}$ & $\mathrm{N} / \mathrm{A}$ & $\mathrm{N} / \mathrm{A}$ \\
\hline 27 & European & Cattle & Diaphragm & 2900.95 \\
\hline
\end{tabular}

Following specimen enumeration muscle supernatant bacterial suspension loop samples were grown as noted above. Corresponding muscle cryostat sections were gram stained following light fixation. There was one less cattle sampling point for the second Beirut slaughterhouse Facility trial. Chi Square comparison of aerobic cattle and sheep muscle microbial counts obtained at initial visits to the Beirut and Baalbek slaughter houses were highly significantly different to the $90^{\text {th }}$ percent probability level $\left(\chi^{2}=3.4225\right.$, degrees of freedom $=1$, alpha $\left.0.10=2.706\right)$.

Table 2: Facility Specimens RODAC plate and muscle microbial isolates collected during initial Beirut slaughter house visit.

\begin{tabular}{|l|l|l|}
\hline Area 1* & & \\
\hline Location & Bacteria $^{\mathrm{a}}$ & $\begin{array}{l}\text { Bacterial Count } \\
\mathrm{cfu} / \mathrm{cm}^{\mathrm{b}}\end{array}$ \\
\hline Floor & Total Aerobic & TMTC \\
\hline Floor & Unidentified & TMTC \\
\hline Wall & Unidentified & 0.5 \\
\hline Area 2* & & \\
\hline Location & Bacteria $^{\mathrm{a}}$ & $\begin{array}{l}\text { Bacterial Count } \\
/ \mathrm{cm}^{\mathrm{b}} \text { cfu }\end{array}$ \\
\hline Wall & Pantoea sp (89.2\%) & 332.5 \\
\hline
\end{tabular}

\begin{tabular}{|l|l|l|}
\hline Wall & Unidentified & 69 \\
\hline Floor & $\begin{array}{l}\text { Chryseomonas luteola } \\
(93.8 \%)\end{array}$ & 119 \\
\hline Floor & Total Aerobic & TMTC \\
\hline Percentage of Muscle Isolates & $\begin{array}{l}\text { Percentage of specific isolates from nine } \\
\text { individual muscle samples }\end{array}$ \\
\hline $\begin{array}{l}\text { Bacterial Species } \\
\text { Isolated }\end{array}$ & 11.1 & \\
\hline $\begin{array}{l}\text { Pasteurella pneumonia } \\
\text { haemolytica }\end{array}$ & 22.2 & \\
\hline Klebsiella oxytoca & 11.1 & \\
\hline Y. enterocolitica & 11.1 & \\
\hline Pantoea spp & 66.7 & \\
\hline Unidentified & \multicolumn{2}{|l|}{} \\
\hline Microbial Enumeration & Average Bacterial Count/ Cm² \\
\hline $\begin{array}{l}\text { Bacterial Species } \\
\text { Isolated }\end{array}$ & 332.5 & \\
\hline Pantoea spp & 119 & \\
\hline Chryseomonas luteola & &
\end{tabular}

Specimens were obtained from two Awassi sheep, one cow from local herds, four Brazilian and two European (French / Holland) imported origin cattle. The percentages of specific bacterial isolates obtained from muscle samples refer to the number of positive cultures obtain from the nine samples taken per muscle sample. Similarly, the average count is that of specific bacterial species isolations obtained at specific slaughterhouse locations. ${ }^{a}$ Bacteria were identified following Gram staining, culture on specific media, API test and streptococcus identification testing (Manero

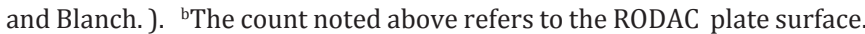

Table 3: Facility specimen RODAC plate and muscle microbial isolates collected during initial Baalbek slaughterhouse visit.

\begin{tabular}{|c|c|c|}
\hline \multicolumn{3}{|l|}{ Area 1* } \\
\hline Location & Bacteria $^{a}$ & $\begin{array}{l}\text { Bacterial Count }{ }^{\mathrm{b}} \mathrm{cfu} / \\
\mathrm{cm}^{2}\end{array}$ \\
\hline Wall & Total Aerobic & TMTC \\
\hline Wall & Salmonella & 0 \\
\hline Floor & Enterococcus spp & 15.6 \\
\hline Floor & E. coli $(96.1 \%)$ & 23.8 \\
\hline Floor & Staphylococcus aureus & 39 \\
\hline \multicolumn{3}{|l|}{ Area $2^{*}$} \\
\hline Location & Bacteria $^{\mathrm{a}}$ & Bacterial Count $\mathrm{t}^{\mathrm{b}} / \mathrm{cm}^{2}$ \\
\hline Wall & Total Aerobic & TMTC \\
\hline Wall & Y. pseudotuberculosis & 0.8 \\
\hline Floor & Enterococcus spp & 49.5 \\
\hline Floor & Salmonella spp & 0 \\
\hline Floor & Staphylococcus aureus & 12 \\
\hline \multicolumn{3}{|c|}{ Percentage of Muscle Isolates } \\
\hline $\begin{array}{l}\text { Bacterial Species } \\
\text { Isolated }\end{array}$ & \multicolumn{2}{|c|}{$\begin{array}{l}\text { Percentage of specific isolates from nine } \\
\text { individual muscle samples }\end{array}$} \\
\hline E. coli & 11 & \\
\hline Staphylococcus spp & 55.6 & \\
\hline Streptococcus spp. & 0 & \\
\hline
\end{tabular}




\begin{tabular}{|l|l|l|}
\hline Y. pseudotuberculosis & 66.7 & \\
\hline Others & 0 & \\
\hline Unidentified & 0 & \\
\hline Microbial Enumeration & \\
\hline $\begin{array}{l}\text { Bacterial Species } \\
\text { Isolated }\end{array}$ & Average Bacterial Count/ Cm ${ }^{2}$ \\
\hline E. coli & 23.8 & \\
\hline Staphylococcus spp & 25.5 & \\
\hline Enterococcus spp & 57.3 & \\
\hline Y.pseudotuberculosis & 0.8 & \\
\hline
\end{tabular}

Specimens were obtained from two Awassi sheep, four cattle from local herds and three imported Brazilian origin cattle. The percentages of specific bacterial isolates obtained from muscle samples refer to the number of positive cultures obtain from the nine samples taken per muscle sample. Similarly, the average count is that of specific bacterial species isolations obtained at specific slaughterhouse locations. ${ }^{a}$ Bacteria were identified following Gram staining, culture on specific media, API test and streptococcus identification testing (Manero and Blanch, 1999.

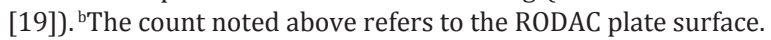

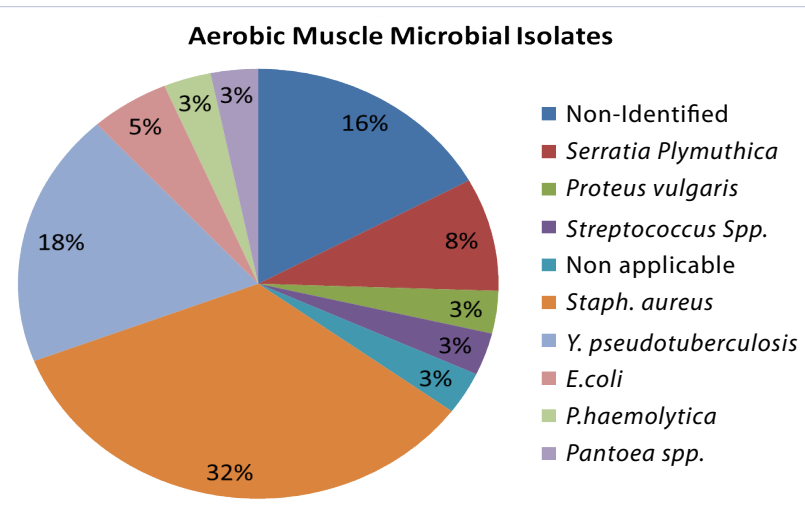

Figure 5: Aerobic microbial isolates from muscle specimens obtained from hot carcasses in Baalbek and Beirut slaughterhouse facilities. The most frequently isolated bacterial species, as depicted by differences in shading intensity and label (above) were Staphylococcus aureus (32 $\%$ ), followed by Yersinia pseudotuberculosis (18\%), Proteus vulgaris (8 $\%)$, Klebsiella oxytoca (8 \%), Escherichia coli (5\%), Pasteurella pneumotropica / haemolytica (3\%), Serratia plymuthica (3\%), Pantoea species (3\%) and Streptococcus species (3\%) A sizable percentage (16\%) of microbial isolates was not identified using these methods.

At our second visit to the Beirut slaughterhouse a different spectrum of bacteria were isolated that exhibited highly represented in species isolated from representative muscle specimens. These included Staphylococcus aureus (87.50\%), Klebsiella oxytoca (12.50\%), Streptococcus species (12.50\%), Proteus vulgaris (37.50\%), Escherichia coli (12.50\%) and Serratia plymuthica $(12.50 \%)$ ( $\mathrm{n}=8$ muscle specimens). The level of microbial isolation found in the Beirut slaughterhouse at our second visit decreased in comparison to the first visit. At the second visit these species included Staphylococcus aureus, Klebsiella oxytoca, Streptococcus species, and Citrobacter species a (enumerated at $3.81,1.68,3.15$ and 28.60 per $\mathrm{cm}^{2}$ muscle specimen, respectively) [Table 2].
Enumeration of the muscle specimen supernatant cultures on our sole visit to the Baalbek Slaughterhouse detected a broad group of microbial species [Table 3]. There were elevated microbial species percentages detected in carcass muscle specimens from this site also. Escherichia coli (11.10\%), Staphylococcus (55.55\%) and Yersinia pseudotuberculosis $(66.66$ $\%)$ populations were frequently isolated at levels in excess of 100 colonies per $\mathrm{cm}^{2}$ muscle specimen $(\mathrm{n}=9)$. The average bacterial counts per $\mathrm{cm}^{2}$ muscle specimen were lower in the Baalbek Facility than in carcass muscles being slaughtered and processed in the Beirut Facility [Tables 2,3]. Microbial species detected in the Baalbak slaughterhouse included Escherichia coli, Staphylococcus aureus, Enterococcus species and Yersinia pseudotuberculosis (enumerated at 23.8, 25.2, 57.3 and 0.8 colonies per $\mathrm{cm}^{2}$ muscle specimen sampled respectively). A chi square comparison of aerobic cattle and sheep muscle microbial counts obtained at our initial visits to the Beirut and Baalbek Slaughterhouses were highly significantly different to the $90^{\text {th }}$ percent probability level $\left(\chi^{2}=3.4225\right.$, degrees of freedom $=1$, alpha $\left.0.10=2.706\right) . \mathrm{We}$ did not isolate any Salmonella or Streptococcus species, and had no unidentified bacterial species in muscle supernatant cultures originating from the Bekka'a slaughterhouse facility samples.

\section{Environmental microbiology-slaughter houses}

Our in-house RODAC plates [Figure 1] allowed us to obtain direct surface impressions of slaughterhouse walls and flooring. Upon incubation of these plates we were able to detect a variety of different microbial species in both slaughterhouse facilities. The facility walls and floors were sampled during slaughtering periods at specific, representative locations along the slaughtering line and are identified as indicated in Figures 2,3. Both Baalbek facility areas one and two [Figure 2 and Table 3] surveyed exhibited too many aerobic colonies to count on the wall sampling sites. Subsequent culture and enumeration of those specimens determined that Yersinia pseudotuberculosis (0.8 cfu per $\mathrm{cm}^{2}$ RODAC plate surface) was present on walls. Higher levels of Escherichia coli (23.8 cfu per $\mathrm{cm}^{2}$ RODAC plate surface obtained from the area 1 floor site); Enterococcus species (15.6 $\mathrm{cfu}$ per $\mathrm{cm}^{2}$ RODAC plate surface in area one, and $49.5 \mathrm{cfu}$ per $\mathrm{cm}^{2}$ RODAC plate surface in area two floor sites); and Staphylococcus aureus (39.0 cfu per $\mathrm{cm}^{2}$ RODAC plate surface in area one, and $12.0 \mathrm{cfu}$ per $\mathrm{cm}^{2}$ RODAC plate surface in area two floor sites) being the main species represented in the Bekaa Valley facility [Table 3]. No Salmonella species organisms were detected on either side of the facility.

The first visit to the Beirut slaughterhouse facility yielded aerobic RODAC cultures from areas one and two floor sampling sites [Figure 3] that were enumerated as too many to count. Both floor and wall sites located on areas 1 and 2 within the Beirut slaughterhouse yielded a large number of organisms that were not able to identified with these methods [Table 2]. Bacterial counts were slightly lower in area two, where cattle were slaughtered, which when enumerated yielded Pantoea species and Chryseomonas luteola colonies (332.5 per $\mathrm{cm}^{2}$ of RODAC wall sampling plate surface, and $119 \mathrm{cfu}$ per $\mathrm{cm}^{2}$ RODAC floor sampling plate surface, respectively). There were a substantial 
number of unidentified microbial species ( 69.0 bacterial colony forming units per $\mathrm{cm}^{2}$ of RODAC plate surface) isolated from area one walls and floors, and area two walls (0.5, too many to count, and $69 \mathrm{cfu}$ per $\mathrm{cm}^{2}$ RODAC plate surface, respectively) [Table 2]. For our second visit to the Beirut slaughterhouse facility we still detected large numbers of bacterial colonies from the floor of area one (too many to count), but there were fewer overall numbers of colonies enumerated with a different set of microbial organisms. Area one of the Beirut slaughterhouse contained Citrobacter species and Staphylococcus aureus (28.6 and $7.2 \mathrm{cfu}$ per $\mathrm{cm}^{2}$ RODAC plate surface respectively) which represented decreased isolation levels compared to those obtained following the first visit. Area two of the Beirut slaughterhouse on the second visit contained lower aerobic total enumerates (38.8 $\mathrm{cfu}$ per $\mathrm{cm}^{2}$ RODAC plate surface) and fewer colony isolations (Klebsiella oxytoca, Staphylococcus aureus, and Streptococcus species at 2.68 cfu, $0.42 \mathrm{cfu}$ and $3.5 \mathrm{cfu}$ per $\mathrm{cm}^{2}$ RODAC plate surface, respectively.

\section{Discussion}

\section{Elevated microbial counts present in Beirut location}

Our study suggests that there is significant bacterial contamination of slaughterhouse environments in both settings, but that contaminant levels were higher initially in the Beirut slaughterhouse in comparison to that in the more rural Bekaa Valley location. This is the first published study of this type in Lebanon. Accordingly, this is the first report of bacterial loads present in red meat animal slaughterhouse environments and can serve as baseline data from which to develop standard operating procedures that are conceptually in compliance with appropriate food safety management systems such as Hazard and Analysis Critical Control Points (HACCP), Good Manufacturing Practice (GMP) and Good Hygiene Practice (GHP) [21]. Because this study has provided location and facility-specific microbial isolation frequencies and speciation spectra there is now a basis from which to work to improve food safety, security, and trade [22] by identifying Critical Control Points from which to decrease microbial contamination of muscle destined for human meat consumption that is not usually chilled, singed or disinfected as a part of the initial slaughterhouse carcass processing system, and which may be sold through wet markets in rural areas especially for fast consumption.

There are currently no published microbial isolation performance data during food animal slaughter at the beginning of the meat food in Lebanon. In addition, there are no current Lebanese governmental microbial performance criteria and surveillance systems for red meat as have been developed in the US and EU [23-25]. In particular, health risk assessment involves four analytical steps consisting of hazard identification followed by characterization of the hazard, human and animal exposure, and quantitative characterization of the risk of adverse effects, and all four steps are important preludes to decision making [26] and programmatic development. A recent USA Agency for International Development Lebanon Industry Value Chain Development Project has focused on flowers, vegetable and fruit produce but not meat [27]. In the US food safety relies on federal oversight of ongoing slaughterhouse microbial surveillance and pathogen reduction efforts through HACCP $[21,26]$. A developing and instituting risk analysis criterion for food safety control is imperative in this process. Measures such as food safety objectives (which set maximum frequencies or occurrences of food hazards at consumption), performance objectives (maximum frequencies or occurrences of food hazards at specified steps in the food chain), and performance criterion ( the effects achieved by applying specific control measures in a process contributing to the performance or food safety objective) are essential in identifying individual steps in a process by which hazard reduction can be reduced to levels that are consistent with overall food safety targets in the food production supply chain $[21,26]$. Individual operating managers, organizations and governments can develop and monitor compliance with these criterion and objectives through operations management, by instituting control measures, activities that prevent or eliminate food safety hazards or reduce the hazard to an acceptable level (acceptable level of protection) [21]. Ascertaining risk includes that posed through public health infectious disease, contaminations, intoxications; hygiene standards, which are necessary but not sufficient, and which are stipulated in the European Food Safety Authority Council Regulation No. 178/2002 and European Union Decision 2001/471/EC, but are not dependent on pathogen reduction as a goal; and, financial risk associated with food spoilage, loss and waste during business operations $[21,28,29]$. One goal of this research study has been to identify critical points in the red meat slaughtering process at which intervention can reduce bacterial loads associated with human and animal disease pathogens as well as food spoilage. These interventions can potentially include actions such as setting microbial isolation level criterion, pathogen control guidelines, codes of hygiene, developing external quality assurance relationships, training and educational programs for slaughterhouse employees and syndicate members, and infrastructural legislative change low and middle income countries that effectively set and enforce food safety standards [21].

The methods used in this manuscript to isolate carcass and facility microbes may represent a technical improvement on current microbial surveillance practices. In addition to direct sampling of slaughterhouse surfaces we utilized sterile sampling technique for muscle sites from largely interior muscle bundle locations, and modified traditional sterile muscle cell culture techniques for use in obtaining microbial population's present within the carcass. In most instances carcass microbial loads are assessed in HAACP testing using wet or dry swabs of muscle surfaces. We believe that using this technique is a useful way to also assess microbial populations that might be present deeper in the muscle in addition to those that have settled on the carcass surface during processing.

One important factor affecting differences in bacterial aerobic counts between the two facilities could be differences in locale and hygienic practices [30]. Imported cattle predominantly, but some sheep and goats also, are housed in small groups and lots within the outdoor corrals immediately adjacent to the Beirut 
facility. Because the Beirut slaughterhouse is located adjacent to the Port of Beirut, the Mediterranean Sea, and several large highways with high traffic loads, this location may be associated with increased dust, particulate levels and microbial populations with extensive opportunities for exposure to additional bacterial agents [31-33]. Lebanon receives anthropogenic and natural aerosols from central Europe, Asia and Africa [31] with increased aerosolization noted in spatial locations that are near highways [32]. In a recent study systemic measures of bacterial and fungal levels in common venues determined that six parameters influenced these levels: 1) microenvironment type; 2) sampling time; 3) fungal agar type, 4) seasonal variation, 5) facility location and 6) summer study periods. [33]. Total bacterial aerosol counts were generally between 10 to $103 \mathrm{cfu}$ per $\mathrm{cm}^{3}$; this country set a maximum indoor bioaerosol concentration of $800 \mathrm{cfu}$ per $\mathrm{cm}^{3}$, which may have been exceeded in the study described herein. In comparison, the Baalbek slaughterhouse was supported by a smaller number of people, appeared to have less foot traffic within the slaughterhouse and was situated in a low traffic rural landscape. In most instances herds were not housed for longer time periods on site, but were brought to the slaughterhouse in small numbers by individual farmers and herdsmen and were killed and processed immediately upon arrival.

\section{Slaughterhouse microbiome}

The slaughterhouse surface Microbiome most likely reflects combined contributions from several different sources [29]. The animals themselves will carry microbial populations on their wool, hair and skin as well as internal enteric microflora that can be dispersed onto slaughterhouse facility surfaces during the sequence of slaughter and processing unless preventative steps are instituted. Human staffing and visitors walking within the slaughterhouse facilities constitute additional contaminant sources as can the water, equipment and products used to cleanse and disinfect the facility. Bio aerosols can be deposited through open doors to each facility and are most likely derived from local geographic sources, including small particles of less than 5 micrometers in size which can be easily inhaled into alveolar spaces [31-33]. Previous studies have documented that the major causes of microbial contamination on beef [29] and swine $[34,35]$ fecal, pharyngeal and environmental. Specific steps for HACCP and GMP compliance were identified in carcass slaughtering and dressing that were associated with pathogen reduction, including scalding and singeing [29]. Critical control points in slaughter practices that were identified by these investigators included scalding, hot water washes. Use of clean room operations is recommended for circumanal incision and removal of the intestines, excision of the tongue, pharynx and tonsils, postmortem inspection procedures and deboning of the head [35]. Performance criteria in these instances are measured by quantitating the presence of E. coli, Enterobacteriaceae species, Salmonella species, overall aerobic mesophilic culture counts and aerobic resuscitation counts [33,35].

\section{Animal health and farm certification}

Promoting robust food animal health at farms and ranches of origin is the building block upon which all of the subsequent steps are developed. Standardized, attentive and complete animal nutrition, husbandry and veterinary preventative care practices can go a long way towards alleviating animal stress and disease $[23,24]$, including subclinical and/ or asymptomatic infection or carcass population with microbes that might be disadvantageous for human and animal health. Practices to avoid might include persistent subclinical antibiotic treatment which allows most food animals to exhibit faster and higher feed conversion rates thus becoming more productive more quickly, but which also predisposes them towards carrying antibiotic-resistant strains of different bacterial populations [20,23]. Regular deworming of food animals might decrease levels of parasitism observed in animals coming to slaughter, including those species that are infectious to humans such as Taenia saginata in cattle (and sometimes sheep and goats) for which humans are a definitive host, and Taenia ovis in sheep muscle, Taenia hydatigena in liver and Taenia multiceps of the muscle, brain, subcutis and eye, especially $[36,37]$. These parasites are not always visually identified using organospecific postmortem evaluation with early prepatent infections. Small ruminants, unlike cattle, have very few antiparasitic drugs that are specifically approved for these species. More practically, a regional hostile incursion prevents provision of adequate veterinary care, regular animal husbandry, and interrupts needed supply chains for drug delivery.

\section{Bacterial cultivation, speciation and identification}

Animals processed through slaughterhouse processes without preventive intervention steps will be contaminated with environmental microbes. Soil sources may contain up to 1010 microbial cells per gram of complex soil material in total microbial populations that include bacteria, viruses, fungi, nematodes and protozoa [38,39]. However, $99 \%$ of these microbes are viable but not cultivatable [38] and so identifying organisms using predominantly cultural methods may be sampling a small subset of the actual microbial population present in any given soil [38-40]. Microbial community settings, such as biofilms, are characterized by key cell-cell communication networks between cells, metabolite trading and resource competition [41] which together promote bacterial adaptation to environmental conditions - resulting in microbial ecological niche development, and strain evolution [41]. These cellular networks are difficult to replicate in monolayer and/or pure cultures, resulting in growth failure [41]. There are also many species of bacteria for which clearly supportive culture conditions containing essential and productive nutrients have not been defined, for which additional strategies (e.g. diffusion chamber culture, bioreactors, prolonged cultivation, alternative carbon sources utilization) are necessary for resuscitation of these strains from environmental specimens [41]. Bacterial speciation has traditionally relied on assumption of underlying genetic similarities that result in characteristic appearance and biochemical reaction phenotypes [42]. A bacterial species has traditionally been defined using DNA-DNA hybridization techniques with a- 70\% hybrid binding properties and less than 5\% difference in DNA melting temperature; and, b) $97 \%$ plus 16 s ribosomal RNA gene sequence identity serving 
as the traditional definition of bacterial species taxonomy [43]. It is becoming increasingly clear, however, that the concept of microbial species may be overly broad, including microbes that may be better suited to be placed in other phyla upon taxonomical evaluation, and that biodiversity among microbial species is much larger than previously considered [42]. Genetic exchange occurs in bacteria within and across currently defined species lines, through transformation (absorption of genetic material present in the local environment), transduction (transfer of genetic material mediated by viruses) and conjugation (transfer of genetic material form one microbe to another) [42]. As a result, microbial species in particular are sometimes considered to be "fuzzy", with individual strains becoming divergent enough that they are not detected using classical microbiological techniques [42]. In turn, genetic variation and changes in nucleic acid expression underlying biochemical reactions that form the basis, along with culture phenotype, of classical microbial species screening methodology become inaccurate and less able to clearly identify or distinguish isolates [43]. Even within microbes that have been identified as being members of a given species there are distinct variations in metabolic capacity and ecological niche that might impact phenotypic analysis and screening using classical methods [43]. Additional methodologies, including whole cell fatty acid chemotaxis analysis, nucleic acid typing methods and 16s rRNA gene sequence analysis are typically used to fully identify representatives of different taxonomic gene clusters, determine the cladistic phylogenetic classification, and then perform DNA-DNA hybridization for speciation [43].

Mesophilic Enterobacteriaceae spp. is not recommended as indicator organisms to use as a part of HACCP programs [29,44]. These experiments did not result in isolation of Salmonella species, which has been classically isolated in pork and poultry industry food chains $[34,45]$. While it is possible that low levels of this organism are present in these settings, growths in some species are suppressed by cold temperatures. Accordingly, it is also possible that isolation of ubiquitous psychrotrophic Enterobacteriacea spp. that originate in soil, pasture, hide and wool, will both outnumber thermotropic Enterobacteriaceae spp. such as some E. coli and Salmonella spp and will grow more rapidly when muscle specimens are incubated at $30-37^{\circ} \mathrm{C}[44]$. The result will be overgrowth of psychotropic species that may obscure the presence of temperature sensitive fecal contaminants $[29,44]$.

When the results of this study are compared to published criterion used in USA and EU it is apparent that microbial organisms are present at levels that exceed current recommended criteria $[21,26,28,29]$. Minced meat should contain no more than $5 \times 10^{6} \mathrm{cfu}$ aerobic bacteria per gm of meat, and /or $500 \mathrm{cfu}$ of nonpathogenic E. coli per gm of meat [45]. Raw sliced beef, poultry and seafood meal should contain no more than $1 \times 10^{5}$ cfu aerobic colonies per gm of meat after being incubated at $300^{\circ} \mathrm{C}$ for 48 hours [45]. The aerobic bacterial plate counts that were obtained from Lebanese slaughterhouses in this study do appear to exceed the nonpathogenic $E$. coli standard for raw meat product noted above in some instances.

\section{Assessing microbial contamination origin}

Soil and water microbial content is classically assessed in part by determining Heterotrophic Plate Count (HPC) to identify nonpathogenic indigenous bacteria that do not pose any threat to human health $[46,47]$. In soil, these populations typically consist of Arthrobacter, Streptomyces species and Pseudomonas species microbes $[38,39,40]$. Species that are important mediators of environmental biological functions include Nitrosomonas, Nitrobacter, Thiobacillus, Actinomycetes, Bacillus, Clostridium, Methanotrophs general, Alcaligena, Rhizobium, Frankia and Agrobacterium [47], most of which were not detected in our study.

Water borne heterotrophic bacteria tend to be gram negative species, and to be present at levels ranging from less than 1 Colony Forming Unit (CFU) per milliliter of water, to $10^{4}$ CFU per milliliter of water $[38,48]$. These counts vary with the temperature, level of organic materials present, and in tap and recreational water, the presence of chlorine and/or other water treatments in place $[38,48]$. We expected to detect microbes that might be present in water supplies used in sanitation control for both facilities; such water washed microbial populations would be those transmitted through direct contact as a result of inadequate sanitation or hygiene, and can be diminished by using copious amounts of water for surface cleaning purposes as well as hand washing $[38-40,48]$. In this instance the quality of the water seems to be less important; however, use of inadequate water quantities in cleaning can result in diarrheal diseases in susceptible populations (typically the young, elderly, and those with predisposing illnesses) as well as non-diarrheal human diseases such as infectious conjunctivitis, and trachoma [39]. It has been suggested that the water heterotrophic plate count itself "may have an inconclusive impact on illnesses in persons who consume water with high heterotrophic counts" [39].

\section{Relationship between current practices, muscle microbial populations, food safety and health}

Hot or pre chilled carcasses are those that have not undergone chilling to slow microbial reproduction and, when performed in a controlled fashion, to shift microbial populations on chilled carcasses as a function of aging muscle into meat $[17,24,23,30]$. The presence of bacterial counts on raw muscle from pre chilled carcasses is reflective of meat safety slaughter process hygiene [23,24]. Additionally, a number of the isolates that were identified in meat and on facility surface cultures in our study have also been associated with meat spoilage, including Klebsiella oxytoca, Pantoea spp, Proteus vulgaris and Escherichia coli $[17,25,30,49,50]$. Neither slaughterhouse had facilities to allow for rapid carcass chilling or meat aging. Although we met government veterinarians in each slaughterhouse setting, it was only in the Baalbek slaughterhouse facility that we observed a stamped emblem placed onto prime carcass cuts after slaughter, and only once. In all cases meat inspection processes, procedures and applications appeared to be less vigorous when compared to the strength of oversight of commercial owners of the carcasses being processed in each facility. 
A number of the microbial isolates that we identified can be considered to be opportunistic bacterial pathogens that affect susceptible human populations, including the elderly, young, those with predisposing conditions [38-40], and increasingly, those experiencing increased stress secondary to displacement. However, the public health significance of these isolates has not been definitively established for healthy adults without predisposing illnesses in the wider population [38]. Classically these organisms are isolated from health facility water distribution pipelines and grow in untreated and potable water [38-40]. Typical isolates included bacterial species such as Pseudomonas, Aeromonas, Klebsiella, Flavobacterium, Enterobacter, Citrobacter, Serratia, Acinetobacter, Proteus and Providencia [38-40]. These organisms are also considered to be indicator bacteria whose presence signifies that it is possible for pathogenic bacteria to be present [38-40]. Further testing is required to determine whether Klebsiella and E. coli species found in samples are of animal or human origin [38-40]. It would also be important to determine if additional microbial hazard classes such as Giardia lamblia and Cryptosporidium parvum protozoa [51], viruses, fungi and prions [39], chemicals and toxins [45] are present within these tissues. In the case of protozoal agents sanitation to decrease exposure to these agents should incorporate large quantities of wash water as both protozoal species have very low infectious doses [38-40,47] In humans this type of viral and/or protozoal enteric disease tends to be seasonal, and is typically experienced at much higher rates in young children who are placed together in settings such as traditional western-style daycare facilities [38-40,47]. It is not known if this type of elevated incidence is also experienced by local susceptible human populations such as refugees and/or their herds for similar reasons, who may be stressed, and placed into temporary housing.

Diarrheal diseases caused by putative slaughterhouse associated Microbiome bacterial populations are important because they can potentially cause human food borne illnesses and inoculate animals and humans, creating individuals with asymptomatic carrier status [23,38-40,45,47]. These include Salmonella, Shigella, and Campylobacter species as well as Vibrio cholerae, cause disease originating in the intestine, are transmitted via fecal - oral ingestion routes and were not isolated in this study. In addition, we isolated and cultured from both muscle specimens and facility surfaces several bacterial genera and species pathogens that have been implicated previously in causing human food borne illnesses in the US and elsewhere [45]. This category also includes Staphylococcus aureus, Enterococcus species, Streptococcus species, Escherichia coli (including all three strain types, enterotoxigenic, enteropathogenic or enterohemorrhagic) and Yersinia pseudotuberculosis which we were able to isolate from muscle and facility surfaces in both locations. Campylobacter species and Yersinia enterocolitica are both associated with fecal contamination in water and food for animals and humans, originating in animal feces, and have been responsible for large food borne illness outbreaks previously that were traced to drinking water ingestion $[35,52,53]$. Yersinia pseudotuberculosis has also become a recognized human pathogen that is known to be distributed worldwide, but has not been well documented in the US or in Lebanon [52,53]. It is especially important as an emerging disease agent because some strains are able to elaborate a cytotoxic that can enhance disease pathogenicity in some people [53].

Finally, as noted for Salmonella spp., true airborne bacterial organisms such as Brucella species (which is endemic in Lebanon), Salmonella typhi, or Bacillus anthracis were not isolated. It is possible that aside from culture masking that these organisms are not present in abundant enough levels in near enough proximity and/or are too difficult to culture without additional methodological steps to be detected by this method [54]. Much higher personnel protective levels (Biosafety Laboratory 3 for Brucella spp. and Bacillus anthracis) for laboratory personnel who might be working with large volumes of agents that are highly infectious and easily aerosolized. In addition, Yersinia enterocolitica can be isolated at levels that are lower than actual prevalence without incorporating a fast enrichment step into microbial culture - based surveillance protocols [55].

\section{Future Directions}

The result of this initial study suggests several possible pathways for additional research in this area. It would be useful to introduce additional molecular tools to assess microbial genetic content in different locales, facilities, and food products within the red meat food chain.

This initial study also suggests that there is fecal contamination of red meat products as well as bacterial species present that can potentially cause spoilage, human food borne illnesses in Syndicate workers, consumers, and animals if such products are fed back to herds of origin. These challenges can be controlled by identifying HACCP control points that affect farm animal and meat transport and providing appropriate veterinary care and husbandry at farms of origin [56-59] that could lead to a newly developed Farm Accreditation [58] program similar to that found in other settings for incoming red meat farm animals prior to slaughter. Physical facility modifications for consideration include changing animal lairage locations and procedures and exit/ entrance traffic patterns for humans and animals for each slaughterhouse. Animal welfare handling, separation of dirty from clean procedures and a viable consistent inspection service at the point of slaughter and carcass dressing would contribute to developing of individual to national surveillance standards and criteria. Processing practices could include incorporating carcass scalding/ hot washes, chilling facilities, and more detailed sanitation procedures to decrease microbial loads, cross contamination and aerosolization. Hazard analysis critical control point development may require a shift in organizational perspective to encourage a culture of collaboration among currently decentralized governmental units managing this process, to empower veterinarians, operations managers, and other governmental officials in the face of private business interests, and to make extensive improvements in physical plant layout (see below), surface materials, cleaning equipment, detergents and disinfectants, and hygiene when coupled with ongoing staff education could decrease some of the microbial contaminants present in each facility. It will also be important to 
develop individual or governmental performance criteria along with physical infrastructure and new legislation to support these processes and projects. Food safety, security and trade should not be considered in isolation - they are closely linked in determining policies for developing and middle income countries, and are important for public health, microbial evolutionary genetics, animal welfare and market development [22].

Finally, there has not been much official emphasis on ameliorating animal fear and discomfort as a part of slaughter practices currently general practices in Lebanon. Halal slaughter can be performed with enhanced animal welfare with training and oversight. Increased attention to implementing improved animal welfare considerations $[60,61]$ through modernized animal handling and facilitative equipment, upgraded slaughter facilities and consideration of the physical layout of the facilities to exert a more calming influence on entering food animals with resultant stress reduction would be beneficial for animals and employees alike. Animals are housed immediately adjacent to where others of their species are being slaughtered may experience increased stress, which is detrimental to animal welfare and could degrade the quality of meat obtained from them. Food animals could experience a "calm death" using Halal methods [61] with decreased disturbance, decreasing the likelihood of direct workplace injuries and microbial aerosolization, and improving the resultant products obtained at the end of this process.

\section{Acknowledgement}

We acknowledge the assistance of Dr. Bilal Hamad through the Office of the Mayor, City of Beirut, in gaining permitted access to the slaughterhouse environments. We also thank Mr. Ali Zeineddine, Assistant Veterinary Technician for the Beirut Slaughterhouse and Mr. Joseph Mouneim, Director of Slaughterhouse Services at the Beirut Slaughterhouse, for their assistance in executing this study, and Ms. Sally Naalbandian for her assistance in sourcing this work.

\section{Declarations}

\section{Conflict of interest}

Dr. Ali Raad is the Supervising Veterinarian for the Northern District Bekka'a Region, but did not select animals or muscles to be sampled, or influence subsequent data collection, assessment and reporting. All other authors have no conflicts of interest that might affect this work.

\section{Ethical approval}

Institutional and Municipal approvals were sought and granted prior to begin this work. Experimental study protocols were reviewed and approved by the American University of Beirut Institutional Animal Care and Use Committee. Entering slaughterhouses and obtaining study samples required prior permission and permitting by the Office of the Mayor, City of Beirut, Beirut Lebanon, and the Ministry of Agriculture, Country of Lebanon. Slaughterhouses in Beirut and in the Bekaa Valley were overseen by Ministry of Agriculture staff, including governmental veterinarians but preceded on different daily schedules with separate staffing.

\section{Grant Support}

This work was supported by the SEED Grant New Faculty Development Program of the Office of the Dean, Faculty of Agriculture and Food Science, American University of Beirut, Beirut, Lebanon.

\section{References}

1. Asmar FR. Climate and agro-ecological zones. In: Country Pasture/ Forage Resource Profiles - Lebanon. Food and Agriculture Organization of the United Nations; 2011. p. 12-16.

2. Asmar FR. Ruminant livestock production systems. In, Country Pasture/Forage Resource Profiles - Lebanon. Food and Agriculture Organization of the United Nations; 2011. p. 17-24.

3. Anonymous. Strengthening veterinary quarantine capacity in Middle East Countries - final regional workshop" Food and Agriculture Organization of the United Nations; 2013.

4. Beuchat LR. Pathogenic microorganisms associated with fresh produce. Journal of Food Protection. 1996; 59(2): 204-216.

5. Alichanidis E, Polychroniadou A. Characteristics of major traditional regional cheese varieties of East-Mediterranean countries: a review. Dairy Science Technology. 2008; 88: 495-510.

6. Saleh I, Zouhairi O, Alwan N, Hawi A, Barbour E, Harakeh S. Antimicrobial resistance and pathogenicity of Escherichia coli isolated from common dairy products in Lebanon. Ann Trop Med Parasitol. 2009; 103(1): 39-52. doi: 10.1179/136485909X384965.

7. Derra FA, Karlsmose S, Monga DP, Mache A, Svendsen CA, Félix B, et al. Occurrence of Listeria spp. in retail meat and dairy products in the area of Addis Abada, Ethiopia. Foodborne Pathog Dis. 2013; 19(6): 577-579. doi: 10.1089/fpd.2012.1361.

8. Vandendriessche F. Meat products in the past, today and in the future. Meat Sci. 2008; 78(1-2): 104-113. doi: 10.1016/j.meatsci.2007.10.003.

9. Gharios MM. Microbiological quality of selected Lebanese fast foods containing meat. MSc Thesis: American University of Beirut. 2003.

10.Atallah S. How well Lebanon is fiscally decentralized? MDF4 Workshop: Empowering Local Government Institutions in the MENA Region Workshop.

11. Dib H. From traditional to modern: Lebanon's food safety regime in transition. Trade and Development Newsletter; 2010. p. 6-9.

12. Peterson J, Garges S, Giovanni M, McInnes P, Wang L, Schloss JA, et al. The NIH Human Microbiome Project, Genome Res. 2009; 19(12): 2317-2323. doi: 10.1101/gr.096651.

13. Shade A, Handelsman J. Beyond the Venn diagram: the hunt for a core microbiome. Environ Microbiol. 2012; 14(1): 4-12. doi: 10.1111/j.1462-2920.2011.02585.x.

14. Budras KD. Bovine mycology. In: Bovine Anatomy An Illustrated Text. Hannover: Schlutersche Gmbh and Company; 2003. 2-28 and 58-62.

15. Jones SJ, Guru A, Singh V, Carpenter B, Calkins CR, Johnson D. Bovine myology and muscle profiling 2004; Available at http://bovine.unl. edu

16. International Commission on Microbiological Specifications for Foods. Meat Products. In: Microorganisms in Foods 8: Use of Data for Assessing Process Control and Product Acceptance. New York: Springer Science and Business Media; 2011. p. 75-133. 
17. Nychas GJ E, Skandamis PN, Tassou CC, Koutsoumanmis KP. Meat spoilage during distribution. Meat Sci. 2008; 78(1-2): 77-89.

18. Tortora GJ, Funke BR, Case CL. Microbiology: An introduction. San Francisco, CA: Benjamin Cummings; 2004. pp. 1-898.

19. Manero A, Blanch AR. Identification of Enterococcus spp. with a biochemical key. Appl Environ Microbiol. 1999; 65(10): 4425-4430.

20. O'Hara CM, Tenover FC, Miller JM. Parallel comparison of accuracy of API 20E, Vitek GNI, MicroScan Walk/Away Rapid ID and Becton Dickinson Cobas Micro ID-E/NF for identification of members of the family Enterobacteriaceae and common gram-negative, non-glucosefermenting bacilli. J Clin Microbiol. 1993; 31(12): 3165- 3169.

21. Gorris LGM. Food safety objective: an integral part of food chain management. Food Control. 2005; 16(9): 801-809.

22. Unnevehr L. Food safety in food security and food trade. Focus 10 2020 Vision for Food, Agriculture and the Environment. 2003.

23. Duffy G, Lynch OA, Cagney C. Tracking emerging zoonotic pathogens from farm to fork. Meat Sci. 2008; 78(1-2): 34-42.

24. Norrung B, Buncic S. Microbial safety of meat in the European Union. Meat Sci. 2008. 78(1-2): 14-24.

25. Hugas M, Tsigarida E. Pros and cons of carcass decontamination: The role of the European Food Safety Authority. Meat Sci. 2008; 78(1-2): 43-52. doi: 10.1016/j.meatsci.2007.09.001.

26. Hathaway S. Harmonization of international requirements under HACCP-based food control systems. Food Control. 1995; 6(5): 267276.

27. USAID/Lebanon Lebanon Industry Value Chain Development Project. Annual Progress Report. 2013.

28. European Food Safety Authority Legal Foundation. Available from: http://www.efsa.eu/en/efsashow/legal.html.

29. McEvoy JM, Sheriudan JJ, Blair IS, McDowell DA. Microbial contamination on beef in relation to hygiene assessment based on criteria used in EU Decision 2001/471/EC. Int J Food Microbiol. 2004; 92(2): 217-225.

30. Aymerich T, Picouet PA, Montfort JM. Decontamination technologies for meat products. Meat Sci. 2008; 78(1-2): 114-129. doi: 10.1016/j. meatsci.2007.07.007

31. Giorgi F, Lionello P. Climate change projections for the Mediterranean region. Global and Planetary Change. 2008; 63(2,3): 90-104.

32. Monn C. Exposure assessment of air pollutants: a review on spatial heterogeneity and indoor/outdoor/personal exposure to suspended particulate matter, nitrogen dioxide and ozone. Atmospheric Environment. 2001; 35(1): 1-32.

33. Jo WK, Seo YJ. Indoor and outdoor bioaerosol levels at recreation facilities, elementary schools and homes. Chemosphere. 2005; 61(11): 1570-1579.

34. Pearce RA, Bolton DJ, Sheridan JJ, McDowell DA, Blair IS, Harrington D. Studies to determine the critical control points in pork slaughter hazard analysis and critical control point systems. Int J Food Microbiol. 2004; 90(3): 331-339.

35. Borch E, Nesbakken T, Christensen H. Hazard identification in swine slaughter with respect to foodborne bacteria. International Journal of Food Microbiology. 1996; 30(1-2): 9-25.

36. Hoenig EP. Taenia tapeworms: their biology, evolution and socioeconomic significance. Microbes Infect. 2002; 4(8): 859-66.
37. Taenia Tapeworm. Available from: www.pet-informed-veterinaryadvice-online.com/Taenia.html.

38. Pepper IL, Josephson KL. Biotic characteristics of the environment. In: Pepper IL, Gerber CP, Brusseau ML. editors. Environmental and Pollution Science. Burlington: Academic Press; 2006. p. 58-77.

39. Gerber CP, Pepper IL. Microbial contaminants. In: Pepper IL, Gerber CP, Brusseau ML. editors. Environmental and Pollution Science. Burlington: Academic Press; 2006. p.144-168.

40. Maier RM. Biological processes affecting contaminant transport and gate. In: Pepper IL, Gerber CP, Brusseau ML. editors. Environmental and Pollution Science. Burlington: Academic Press; 2006. p. 105-120.

41. Alain K, Querellou J. Cultivating the uncultured: limits, advances and future challenges. Extremophiles. 2009; 13(4): 583-594. doi: 10.1007/ s00792-009-0261-3.

42. Malaterre C. Microbial diversity and the "lower limit" problem of biodiversity. Biology and Philosophy. 2013; 28(2): 219-239.

43. Gevers D, Cohan FM, Lawrence JG, Spratt BG, Coenye T, Feil E, et al. Reevaluating prokaryotic species. Nature Reviews-Microbiology. 2005; 3: 733-739.

44. Struijk CB, Mossel DA. Letter to the editor of the international journal of food Microbiolgy. Int J Food Microbiol. 2003; 99(1): 113-114.

45.FDA. Bad Bug Book, foodborne pathogenic microorganisms and natural toxins. 2nd ed. US Food and Drug Administration. 2012.

46. Payment P, Sartory DP, Reasoner DJ. The history and use of HPC in drinking-water quality management. In: Batram J, Cotruvo J, Exneret M. editors. Heterotrophic Plate Counts and Drinking-water Safety - The Significance of HPCs for Water Quality and Human Health. London: IWA Publishing; 2003; p. 20-48.

47. Walworth J, Pepper IL. Physical contaminants. In: Pepper IL, Gerber CP, Brusseau ML. editors. Environmental and Pollution Science. Burlington: Academic Press; 2006. p. 123-131.

48. Bartram J, Pedley S. Microbiological analysis. In: Bartram J, Balance R. editors. Water quality monitoring - a practical guide to the design and implementation of fresh water quality studies and monitoring programs. New York: CRC Press; 1996.

49. Gram L, Ravn L, Raschm M, Bruhn JB, Christensen AB, Givskov M. Food spoilage--interactions between food spoilage bacteria. Int J Food Microbiol. 2002; 78(1-2): 79-97.

50. Borch E, Kant-Muermans ML, Blixt Y. Bacterial spoilage of meat and cured meat products. Int J Food Microbiol. 1996; 33(1): 103-120.

51. Environmental Protection Agency Method 1623.1: Cryptosporidium and Giardia in water by filtration/IMS/FA 2012. Available from http://water.epa.gov/drink/hotline/index.cfm.

52. Long C, Jones TF, Vugia DJ, Scheftel J, Strockbine N, Ryan P, et al. Yersinia pseudotuberculosis and Y. enterocolitica infections, FoodNet, 1996-2007. Emerg Infect Dis. 2010; 16(3): 566-567.

53. Schweer J, Kulkarni D, Kochut A, Pezoldt J, Pisano F, Pils MC, et al. The cytotoxic necrotizing factor of Yersinia pseudotuberculosis (CNF gamma) enhances inflammation and Yop delivery during infection by activation of rho GTPases. PLoS Pathog. 2013; 9(11): e1003746. doi: 10.1371/journal.ppat.1003746.

54. Department of Agriculture, Australian Government. Approved methods for microbiological testing of meat and meat products. 2013.

55. Manafi M, Holzhammer E. Comparison of the Vitek, API20E and Gene 
Track systems for the identification of Yersinia enterocolitica. Lett Appl Microbiol. 1994; 18(2): 90-92.

56. Cullor JS. HACCP (Hazard Analysis Critical Control Points): Is it coming to the dairy? J Dairy Sci. 1997; 80(12): 3449-52.

57. Australian Standard for the Hygienic Production and Transportation of Meat and Meat Products for Human Consumption. Victoria: Csiro Publishing; 2002.

58. European Union cattle farm accreditation scheme (EUCAS), national livestock identification system and individual animal traceability.
2012. Available at http://www.daff.gov.au/aqis/export/meat/ elmer-3/eucas.

59. Grandin T. Assessment of stress during handling and transport. J Anim Sci. 1997; 75(1): 249-257.

60. Grandin T, Regenstein JM. Religious slaughter and animal welfare: a discussion for meat scientists. Meat Focus International. 1994; 115123.

61. Grandin T. Auditing animal welfare at slaughter plants-Review. Meat Sci. 2010; 86(1): 56-65. doi: 10.1016/j.meatsci.2010.04.022. 